\title{
Reporte de caso: nueva técnica para el manejo de hipospadias refractaria en niños
}

\section{Case Report: New Surgical Technique for the Management of Refractory Hypospadias in Children}

\author{
Federico Gaviria Gil ${ }^{1}$ Johana Andrea Benavides ${ }^{2}$ Juan Fernando Saldarriaga ${ }^{1}$ Matilde Henao ${ }^{2}$ \\ ${ }^{1}$ Hospital Pablo Tobón Uribe, Medellin, Colombia \\ 2 Universidad CES, Medellín, Colombia \\ Address for correspondence Johana Andrea Benavides Martinez, \\ Universidad CES, Cl. 10a \#22 - 04, Medellín, Antioquia, Colombia \\ (e-mail: johanainte08@hotmail.com).
}

Urol Colomb 2019;28:92-96.

\section{Resumen \\ Palabras Clave \\ - hipospadias \\ - uretroplastia con injerto \\ - colgajo en varios tiempos \\ - tubos migratorios}

Introducción y Objetivos Las hipospadias son una malformación congénita de la uretra que resulta en un desplazamiento ventral anormal de la uretra. La corrección quirúrgica requiere del adecuado entendimiento de la anatomía ya que el objetivo es crear un pene con una función y apariencia normal. Aún no hay consenso sobre cuál es la mejor técnica quirúrgica, pero el manejo multidisciplinario puede ofrecer alternativas diferentes en casos complejos. Por esa razón, se realizó en conjunto con cirugía plástica un colgajo en varios tiempos que es utilizado generalmente para cubrir defectos de tejidos blandos obteniendo muy buenos resultados.

Materiales y Métodos Se describe el caso de un paciente con antecedente de hipospadias con correción previa fallida a quien se le realizó manejo conjunto con cirugía plástica; realizando un colgajo inguinal en varios tiempos más uretroplastia con injerto de mucosa oral.

Resultados Paciente de 6 años con antecedente de hipospadias con uretroplastias fallidas. Al examen físico presentaba un pene con hipospadias severa y con cicatriz ventral por procedimientos previos que causaba curvatura. Fue valorado por cirugía plástica quien realizo un Colgajo pediculado inguinal en varios tiempos con interposición de material no absorbible. Cinco meses después, se realizó la uretroplastia con injerto de mucosa oral con rotación e inversión de la parte distal del colgajo para reconstruir el techo de la uretra distal.

Conclusiones Las hipospadias son una malformación congénita compleja de corregir que en varias ocasiones requiere de un trabajo multidisciplinario para ofrecerle al paciente otras opciones de manejo con buenos resultados a largo plazo, sobre todo, cuando las técnicas convencionales han fallado.

Introduction Hypospadias is a congenital anomaly of the male urethra that results in abnormal ventral placement of the urethral opening. Surgical correction requires proper understanding of the penis anatomy to create a penis with normal function and appearance. Actually, there is no consensus about the best surgical technique, but received

October 8, 2017

accepted

November 22, 2017

published online

April 24, 2018
DOI https://doi.org/

10.1055/s-0038-1645848.

ISSN 0120-789X.

eISSN 2027-0119.
Copyright (c) 2019, Sociedad Colombiana License terms de Urología. Publicado por Thieme Revinter Publicações Ltda., Rio de Janeiro, Brazil. Todos los derechos reservados. 


\section{Keywords \\ - hypospadias \\ - uretrhoplasty \\ - graf \\ - inguinal flap}

multidiciplinary management may offer different alternatives in complex cases as was done in this case.

Materials and Methods: We describe the case of a patient with a previous failed hypospadias correction who underwent joint management with plastic surgery; making an inguinal flap plus urethroplasty with oral mucosa graft.

Results a 6 years-old patient with history of previous surgical hypospadias correction which failed, consulted at the service. The physical examination showed a penis with severe hypospadias and with ventral scar by previous procedures that caused curvature. It was evaluated by plastic surgery who performed an inguinal pedicle flap by stages with interposition of non-absorbable material. 5 months later, urethroplasty with oral mucosa graft was performed with rotation and inversion of the distal flap to reconstruct the roof of the distal urethra.

Conclusions Hypospadias are a complex congenital malformation. Surgical management is a challenge. In most difficult cases a multidisciplinary approach is necessary.

\section{Introducción}

Las hipospadias son una malformación congénita de la uretra que resulta en un desplazamiento ventral anormal de la uretra. ${ }^{1}$ Se presenta hasta en un $0,3 \%$ a un $0,7 \%$ de los recién nacidos. ${ }^{2,3}$ La corrección quirúrgica requiere del adecuado entendimiento de la anatomía del pene normal y uno hipospádico, ya que el objetivo es crear un pene con una función y apariencia normal. ${ }^{1,4}$

Mientras más proximal sea el defecto más se asocia a la curvatura ${ }^{5}$ y más complejo es el caso. ${ }^{6}$ El único tratamiento es la corrección quirúrgica. ${ }^{7}$ El momento ideal sería a los 18 meses. ${ }^{8}$ Aún no hay consenso sobre cuál es la mejor técnica quirúrgica ${ }^{9}$ ya que su elección depende del momento en el cual se va a corregir la placa uretral, la calidad de la piel y el esponjoso. ${ }^{10}$

En casos donde procedimientos previos han fallado, el manejo multidisciplinario puede ofrecer alternativas diferentes. Por esa razón, se realizó en conjunto con cirugía plástica, un colgajo en varios tiempos con material no absorbible que es utilizado generalmente para cubrir defectos de tejidos blandos obteniendo muy buenos resultados ${ }^{11}$; asociado a la uretroplastia con injerto de mucosa oral para la corrección quirúrgica del defecto que presentaba el paciente ${ }^{12-14}$

\section{Materiales y métodos}

Se describe el caso de un paciente de 6 años con antecedente de hipospadias con corrección previa fallida, a quien se le realizó manejo conjunto con cirugía plástica; realizando un colgajo inguinal en varios tiempos más uretroplastia con injerto de mucosa oral.

\section{Resultados}

Paciente de 6 años con antecedente de hipospadias a quien ya le habían realizado varias uretroplastias según referían sus padres. Las últimas dos con injerto de mucosa oral que se retraía (labio en una ocasión y carrillo izquierdo en la otra). Al examen físico se observaba un pene con hipospadias penoescrotal severa y con cicatriz ventral con retracción del glande por procedimientos previos que causaba curvatura

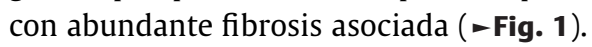

Fue valorado por cirugía plástica quien realizó inicialmente un colgajo pediculado inguinal en varios tiempos con interposición de material no absorbible. Lo que se hizo fue medir la longitud que debería tener el pene y el defecto que se debía cubrir. Se concluyó que se necesitaban $7 \mathrm{~mm}$ para la piel del pene y $14 \mathrm{~mm}$ para la reconstrucción de la uretra (-Fig. 2). Posteriormente se procedió a sacar el colgajo. Se definió tomar el colgajo de la región para-escrotal derecha de una longitud de $21 \mathrm{~mm}$. Se realizó una incisión paralela bilateral y disección para levantarloy dejarlo bipediculado sin sufrimiento ( - Fig. 3). Para eso, se utilizó como recurso, la interposición de un material no reabsorbible (lámina de acetato) para que no cicatrizara y crear la autonomización del colgajo redireccionando el flujo. En ese momento, se le dio de alta al paciente y se le citó para un segundo tiempo.

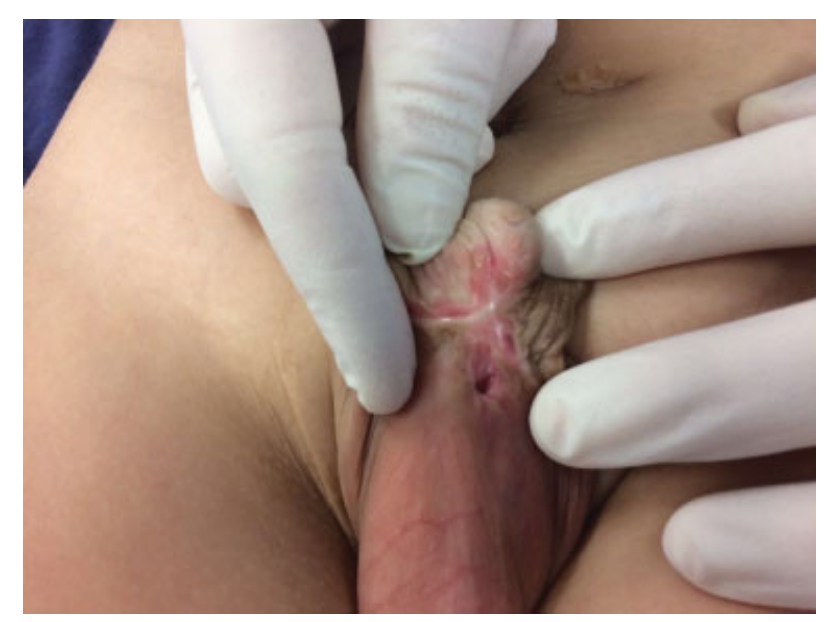

Fig. 1 Hipospadias severa en la base del pene y con cicatriz ventral con retracción del glande. 


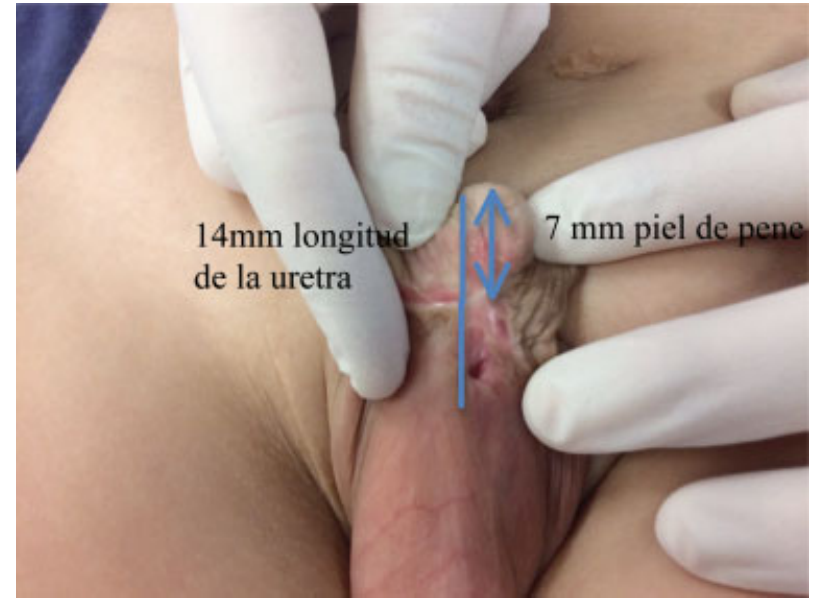

Fig. 2 Medición de la longitud necesaria para poder crear el colgajo.

Cinco meses después, se llevó nuevamente a cirugía y se le realizó uretroplastia con injerto de mucosa oral con rotación e inversión de la parte distal del colgajo para reconstruir el techo de la uretra distal. La técnica consistió inicialmente en resecar el tejido fibrótico ventral y realizar la corrección de la curvatura ventral ( - Fig. 4). Se disecó el meato hipospádico y se calibró con bujía de Hegar 5. Se sacó el injerto de la mucosa oral del carrillo derecho de $4 \times 2 \mathrm{cms}$ y se fijó a los cuerpos cavernosos con vicryl 5-0 (-Fig. 5).

Posteriormente, se realizó rotación y tunelización del colgajo pediculado inguinal derecho prefabricado (-Fig. 6). Se abrió la pseudocapsula del acetato prefabricado y se utilizó como techo de la uretra proximal ( - Fig. 7). Se suturó la pseudocapsula del colgajo a la mucosa del injerto con PDS 5-0. Se pasó sonda de silicona de 10FR. Se realizó inversión de la parte distal del colgajo para reconstruir el techo de la uretra distal y se suturó la piel a la mucosa del injerto con vicryl 5-0 (- Fig. 8).

Finalmente, se remodeló el glande y se creó el neomeato distal con vicryl 5-0- Se cerró la piel del pene y del colgajo (-Figs. 9 y 10). El tiempo quirúrgico fue de 4 horas y 20

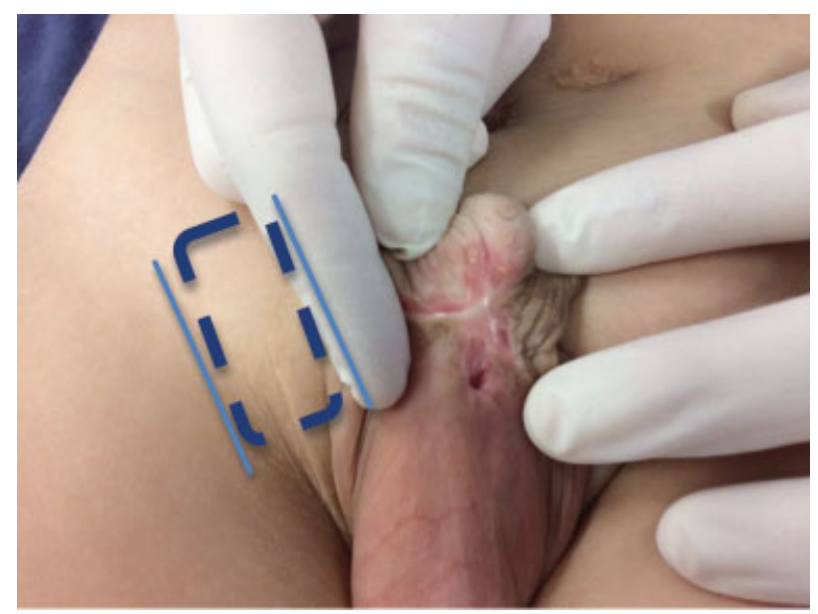

Fig. 3 Línea punteada indica el área seleccionada para realizar el colgajo. Línea continua indican las incisiones paralelas que se hicieron para realizar la disección del colgajo y la interposición del material no absorbible.

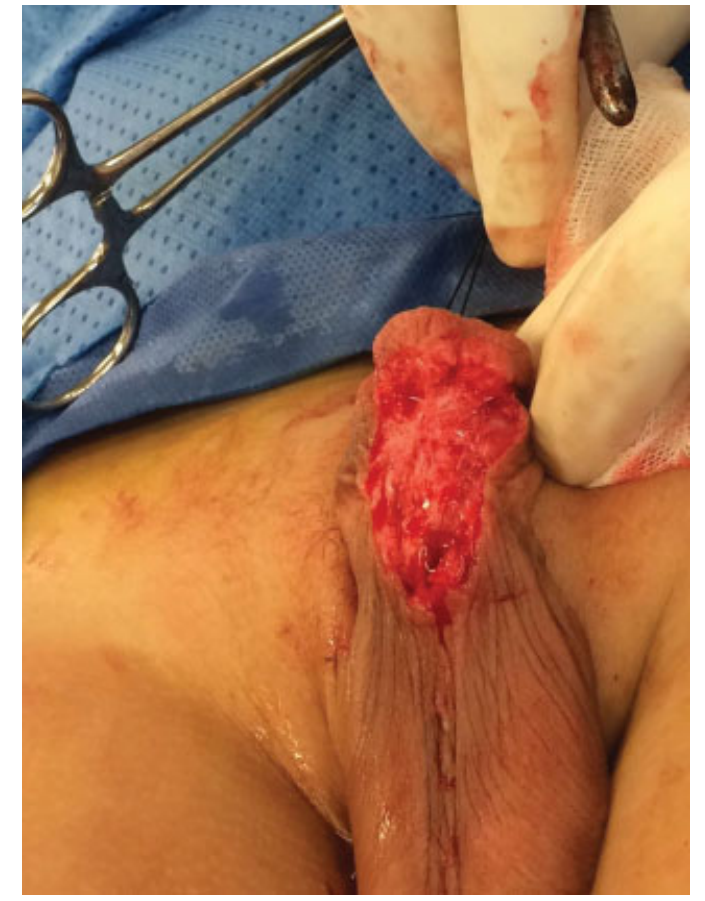

Fig. 4 Resección de tejido fibrótico ventral y la corrección de la curvatura ventral.

minutos. El tiempo de hospitalización fue de 2 días. La sonda se le retiró a las 4 semanas.

El paciente presentó muy buena evolución clínica con corrección completa del defecto y adecuado flujo miccional. $\mathrm{Al}$ año de seguimiento, el paciente continúa asintomático urinario.

\section{Discusión}

El tratamiento para la corrección de las hipospadias es quirúrgico. Existen múltiples variaciones entre las técnicas utilizadas para su manejo. Sin embargo, en todas se hace énfasis en la corrección de la curvatura, la uretroplastia y la reconstrucción de la parte ventral del pene.

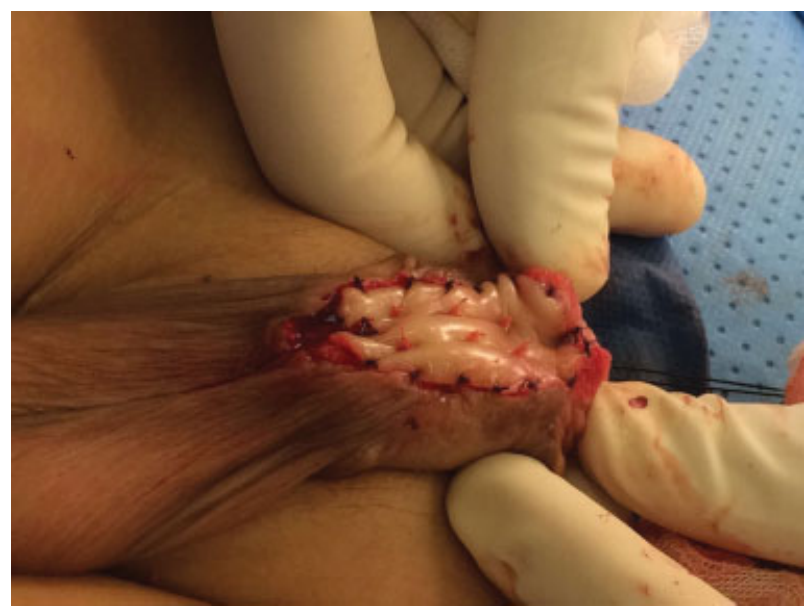

Fig. 5 Injerto de la mucosa oral del carrillo derecho de y fijación a los cuerpos cavernosos con vicryl 5-0. 


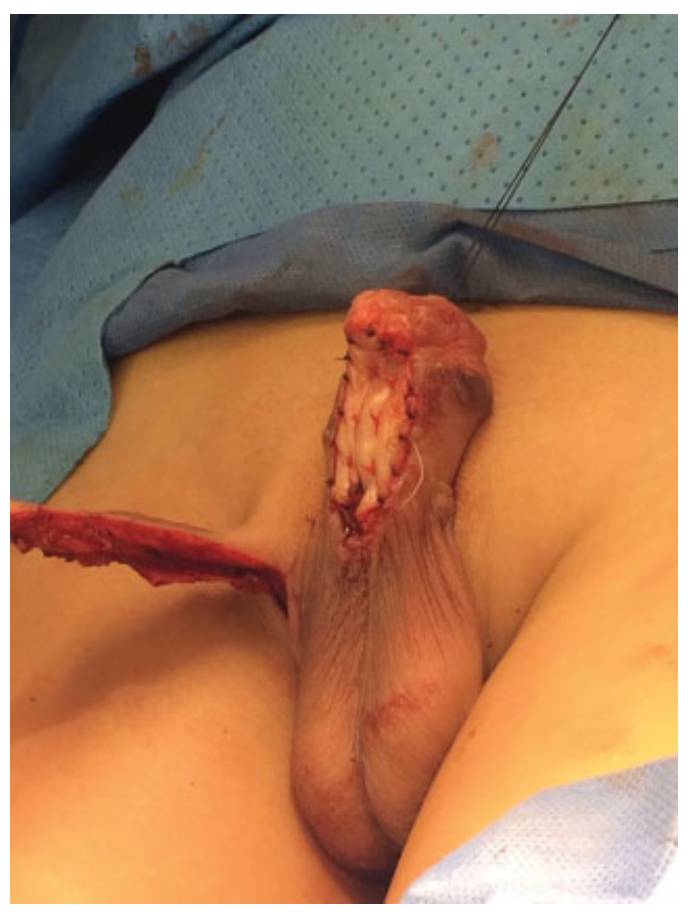

Fig. 6 Tunelización del colgajo.

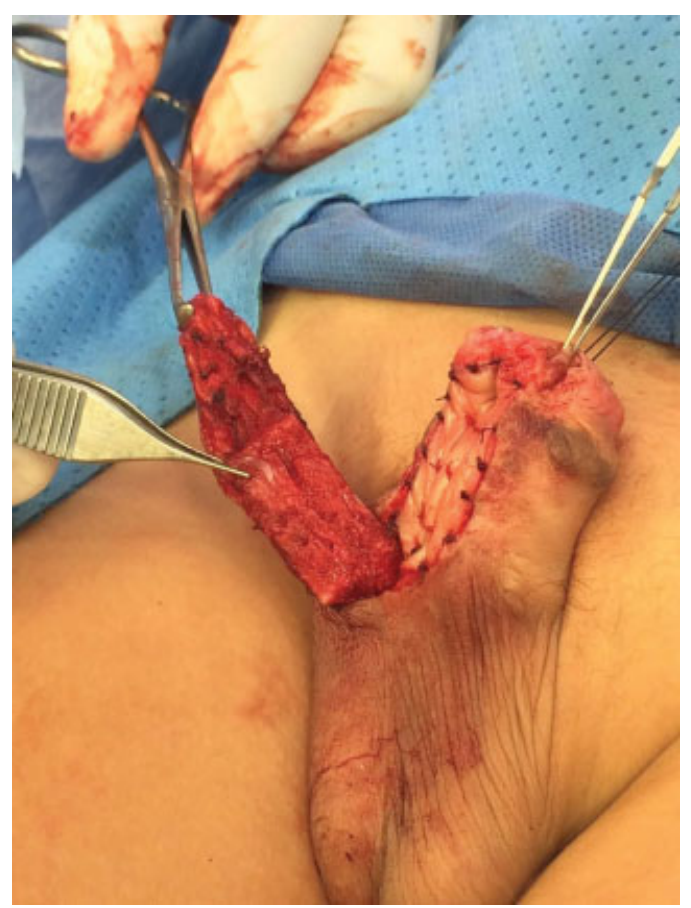

Fig. 7 Apertura de la pseudocapsula del acetato.

Entre las más frecuentes usadas están la tubularización primaria con o sin incisión del plato uretral, ${ }^{12}$ el colgajo transverso prepucial en isla, ${ }^{7,14,15}$ colgajo en isla onlay y la reparación en 2 tiempos.

En el caso expuesto, el paciente presentaba una hipospadias proximal (peno-escrotal). Esa clase, representa uno de los tipos más severos y difíciles de reconstruir y hasta el momento no hay consenso. Entre las técnicas vale la pena mencionar las

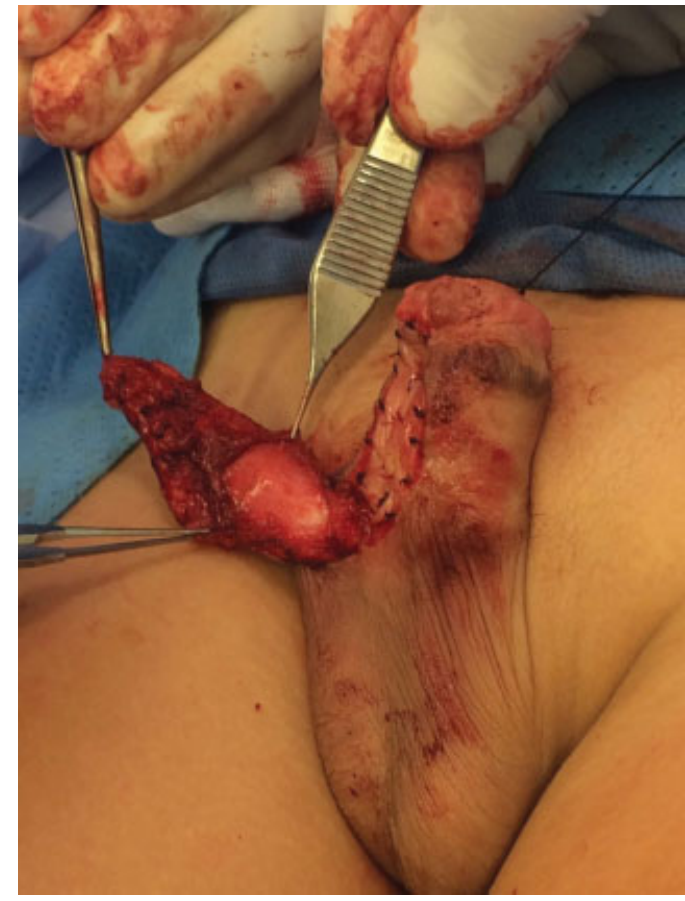

Fig. 8 Fijación al injerto.

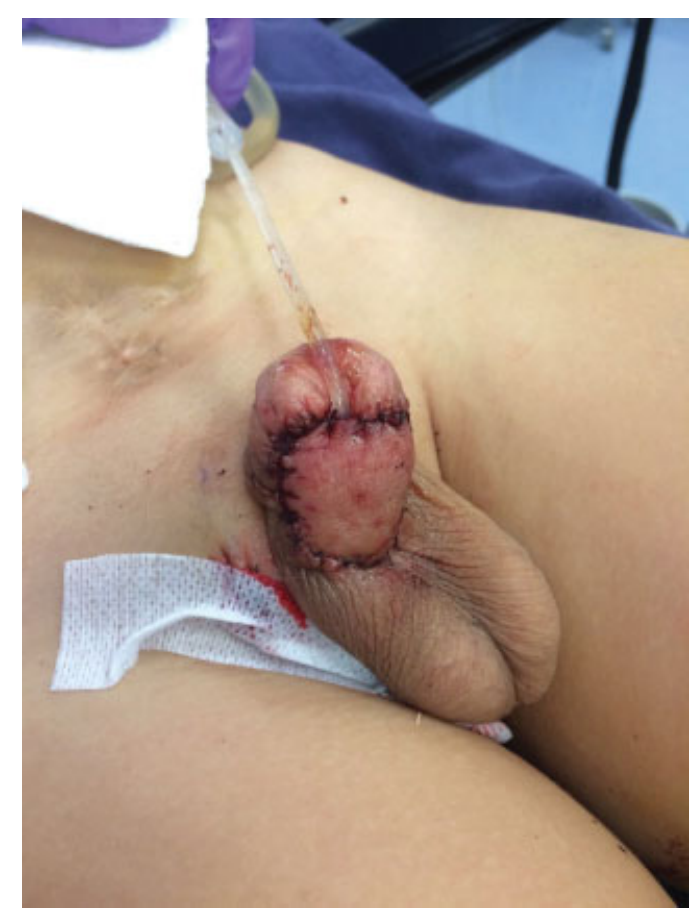

Fig. 9 Aspecto posterior a la reconstrucción.

siguientes: la técnica de snodgrass que consiste en realizar un corte longitudinal en el surco uretral desde el meato ectópico hasta el glande, lo que permite tubulizarlo con menos tensión alrededor de una sonda.

La técnica de Koff que consiste en liberar completamente la uretra peneana de la cara anterior de los cuerpos cavernosos y después trasladarla hacia delante para llevar el orificio uretral a una posición adecuada. La técnica de 


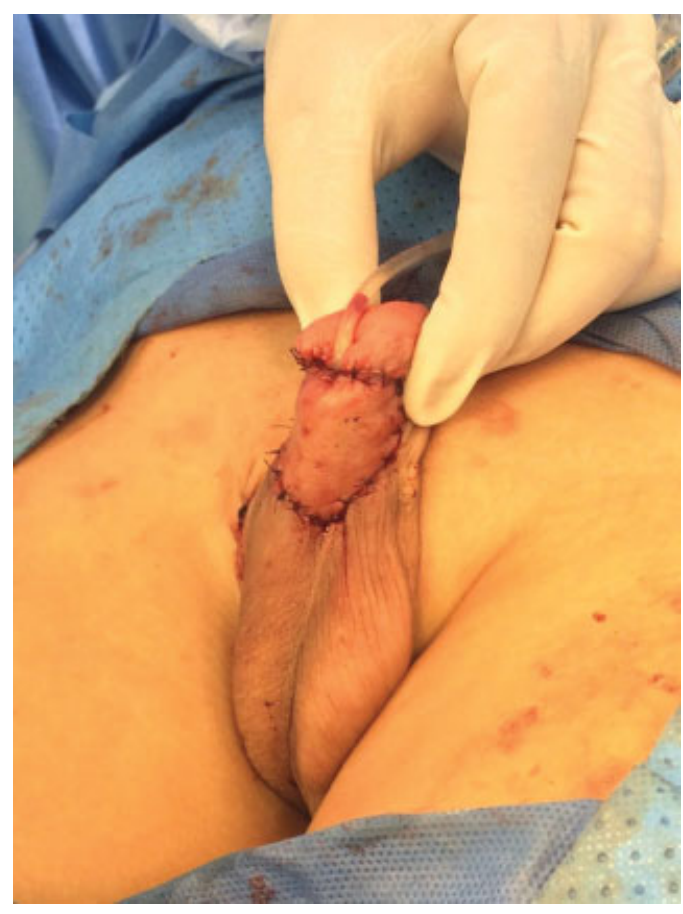

Fig. 10 Aspecto posterior a la reconstrucción.

Koyanagi, en la que el prepucio permanece intacto y se obtiene un solo colgajo cutáneo, que se transpone a nivel ventral a través del pene, realizando un ojal en el pedículo.

Y las técnicas por estadios que se reservan en su gran mayoría para aquellos pacientes que ya han tenido cirugías previas fallidas, en la que se emplea la mucosa oral para la reconstrucción de la neouretra, tratando de preservar lo que quede de la placa uretral. ${ }^{6}$

Las complicaciones ${ }^{16}$ varían de acuerdo a la técnica, experiencia del cirujano y características del paciente. Entre las principales se encuentran la aparición de fístulas, divertículos y la estrechez de la uretra con la subsiguiente necesidad de otras intervenciones.

En el caso presentado, el paciente había presentado varias uretroplastias fallidas por retracción del injerto. Por esa razón se planteó una nueva técnica por estadios en la que se empleó un colgajo además de la mucosa oral.

La técnica descrita aún no ha sido reportada en la literatura para casos de hipospadias, sin embargo, el uso de colgajos tubularizados se podría extrapolar a esos casos, pues es empleada con gran frecuencia para cubrir grandes defectos de cobertura en otras áreas.

\section{Conclusiones}

Las hipospadias son una malformación congénita compleja de corregir que en varias ocasiones requiere de un trabajo multidisciplinario para ofrecerle al paciente otras opciones de manejo con buenos resultados a largo plazo y sobre todo, cuando las técnicas convencionales han fallado.

\begin{abstract}
Financiación
Recursos logísticos y humanos propios.
\end{abstract}

Conflictos de interés

No existen.

\section{Bibliografía}

1 Baskin LS, Ebbers MB. Hypospadias: anatomy, etiology, and technique. J Pediatr Surg 2006;41(03):463-472

2 Baskin LS, Himes K, Colborn T. Hypospadias and endocrine disruption: is there a connection? Environ Health Perspect 2001;109(11):1175-1183

3 Mamoulakis C, Avgenakis G, Gkatzoudi C, et al. Seasonal trends in the prevalence of hypospadias: Aetiological implications. Exp Ther Med 2017;13(06):2960-2968

4 van Rooij IALM, van der Zanden LFM, Brouwers MM, Knoers NVAM, Feitz WFJ, Roeleveld N. Risk factors for different phenotypes of hypospadias: results from a Dutch case-control study. BJU Int 2013;112(01):121-128

5 Mingin G, Baskin LS. Management of chordee in children and young adults. Urol Clin North Am 2002;29(02):277-284, v

6 Keays MA, Dave S. Current hypospadias management: Diagnosis, surgical management, and long-term patient-centred outcomes. Can Urol Assoc J 2017;11(1-2Suppl1):S48-S53

7 Baskin LS, Duckett JW, Ueoka K, Seibold J, Snyder HM III. Changing concepts of hypospadias curvature lead to more onlay island flap procedures. J Urol 1994;151(01):191-196

8 American Academy of Pediatrics. Timing of elective surgery on the genitalia of male children with particular reference to the risks, benefits, and psychological effects of surgery and anesthesia. Pediatrics 1996;97(04):590-594

9 Krull S, Rissmann A, Krause H, et al. Outcome after Hypospadias Repair: Evaluation Using the Hypospadias Objective Penile Evaluation Score. Eur J Pediatr Surg Off 2017

10 Gong EM, Cheng EY. Current challenges with proximal hypospadias: We have a long way to go. J Pediatr Urol 2017;13 (05):457-467

11 Macedo A Jr, Silva MIS, Pompermaier JA, Ottoni SL, de Castro R, Leal da Cruz M. The anterior sagittal transrectal approach (ASTRA) for cases associated with rectal implantation of the urethra: A retrospective review of six cases. J Pediatr Urol 2017;13(06): 613.e1-613.e4

12 Joshi PM, Barbagli G, Batra V, et al. A novel composite two-stage urethroplasty for complex penile strictures: A multicenter experience. Indian J Urol 2017;33(02):155-158

13 McNamara ER, Schaeffer AJ, Logvinenko T, et al. Management of Proximal Hypospadias with 2-Stage Repair: 20-Year Experience. J Urol 2015;194(04):1080-1085

14 Cheng EY, Vemulapalli SN, Kropp BP, et al. Snodgrass hypospadias repair with vascularized dartos flap: the perfect repair for virgin cases of hypospadias? J Urol 2002;168(4 Pt 2):1723-1726, discussion 1726

15 Sedberry-Ross S, Stisser BC, Henderson CG, Rushton HG, Belman AB. Split prepuce in situ onlay hypospadias repair: 17 years of experience. J Urol 2007;178(4 Pt 2):1663-1667

16 Schneuer FJ, Holland AJA, Pereira G, Bower C, Nassar N. Prevalence, repairs and complications of hypospadias: an Australian population-based study. Arch Dis Child 2015;100 (11):1038-1043 